\title{
Safety Performance of Airborne Separation: Preliminary Baseline Testing
}

\author{
Maria C. Consiglio ${ }^{1}$, Sherwood T. Hoadley ${ }^{2}$, David J. Wing ${ }^{3}$, and Brian T. Baxley ${ }^{4}$ \\ NASA Langley Research Center, Hampton, Virginia, 23681
}

\begin{abstract}
The Safety Performance of Airborne Separation (SPAS) study is a suite of Monte Carlo simulation experiments designed to analyze and quantify safety behavior of airborne separation. This paper presents results of preliminary baseline testing. The preliminary baseline scenario is designed to be very challenging, consisting of randomized routes in generic high-density airspace in which all aircraft are constrained to the same flight level. Sustained traffic density is varied from approximately 3 to 15 aircraft per 10,000 square miles, approximating up to about 5 times today's traffic density in a typical sector. Research at high traffic densities and at multiple flight levels are planned within the next two years. Basic safety metrics for aircraft separation are collected and analyzed. During the progression of experiments, various errors, uncertainties, delays, and other variables potentially impacting system safety will be incrementally introduced to analyze the effect on safety of the individual factors as well as their interaction and collective effect. In this paper we report the results of the first experiment that addresses the preliminary baseline condition tested over a range of traffic densities. Early results at five times the typical traffic density in today's NAS indicate that, under the assumptions of this study, airborne separation can be safely performed. In addition, we report on initial observations from an exploration of four additional factors tested at a single traffic density: broadcast surveillance signal interference, extent of intent sharing, pilot delay, and wind prediction error.
\end{abstract}

\section{Nomenclature}

$\begin{array}{ll}\text { ACES } & =\text { Airspace Concept Evaluation System } \\ \text { ADS-B } & =\text { Automatic Dependence Surveillance-Broadcast } \\ \text { AOP } & =\text { Autonomous Operations Planner } \\ \text { ARTCC } & =\text { Air Route Traffic Control Center } \\ \text { ASAS } & =\text { Airborne Separation Assistance System } \\ \text { ASTOR } & =\text { Aircraft Simulation for Traffic Operations Research } \\ \text { ATOL } & =\text { Air Traffic Operations Laboratory } \\ \text { ATOS } & =\text { Airspace \& Traffic Operations Simulation } \\ \text { CD } & =\text { Conflict Detection } \\ \text { CR } & =\text { Conflict Resolution } \\ \text { ETMS } & =\text { Enhanced Traffic Management System } \\ \text { JPDO } & =\text { Joint Planning \& Development Office } \\ \text { LOS } & =\text { Loss of Separation } \\ \text { NAS } & =\text { National Airspace System } \\ \text { NM } & =\text { Nautical Miles } \\ \text { PM } & =\text { Pilot Model } \\ \text { RTCA } & =\text { RTCA, Inc. A non-profit aviation standards setting organization. } \\ \text { SPAS } & =\text { Safety Performance of Airborne Separation }\end{array}$

\footnotetext{
${ }^{1}$ Principal Investigator, Separation Assurance, Crew Systems and Aviation Operations Branch, AIAA Member

${ }^{2}$ Senior Research Engineer, Crew Systems and Aviation Operations Branch, MS152

${ }^{3}$ Senior Research Engineer, Crew Systems and Aviation Operations Branch, MS152

${ }^{4}$ Senior Research Engineer, Crew Systems and Aviation Operations Branch, MS152. AIAA Senior Member
} 


\section{Introduction}

$\mathrm{T}$ he Joint Planning and Development Office (JPDO), lead organization for the development and evaluation of the Next Generation Air Transportation System (NextGen) concept, has directed that understanding the impact of new operational procedures and capabilities on the safe operation of the National Airspace System (NAS) is of foremost importance. The NextGen Concept of Operations ${ }^{1}$ incorporates various types of Airborne Separation Assistance System (ASAS) applications. ASAS applications, as defined by the FAA/Eurocontrol ${ }^{2}$ Action Plan 1, include the categories of situational awareness, spacing, delegated separation, and full responsibility for selfseparation. Before procedures are written and the roles of humans and automation are identified, it is necessary to evaluate the safety performance of these applications.

Airborne separation is enabled by advanced decision-aiding automation integrated into aircraft avionics. The automation is designed to detect conflicts between aircraft, help pilots resolve them, and provide conflictpreventative support for conflict-free maneuvering. These decision aides rely on broadcast surveillance information that includes aircraft velocity vectors and limited flight plan information. To assess safety, it is imperative to understand the effects that changes in traffic levels, information accuracy, prediction errors, datalink range and content, and other uncertainties and variables may have on the distributed automated systems. In particular, the implications of such conditions to the safe operation of NextGen need to be evaluated with comprehensive models that consider all separation assurance applications.

The Safety Performance of Airborne Separation (SPAS) simulation study is designed to investigate the effect of traffic demand on safety performance of distributed airborne separation in the presence of various errors, uncertainties, delays, and other variables potentially impacting system safety. The study is comprised of a series of simulation experiments that progressively characterize and quantify safety in en route airspace using established criteria and safety metrics. The intention is to develop a broad set of generalizable safety performance data for use in safety analyses and for eventual comparison with similar studies focused on ground-based separation.

The primary goal of this preliminary baseline phase of the SPAS study is to develop an initial understanding of the safety performance of airborne separation in a baseline condition as traffic level is significantly increased. An additional goal is to collect initial exploratory data on the impact of several safety-impacting variables to help guide more detailed experimentation planned for later phases. The experiment is conducted in a distributed batch simulation in the Air Traffic Operations Lab (ATOL) of the NASA Langley Research Center. The Autonomous Operations Planner (AOP), an established research software implementation of ASAS automation, is used to provide the airborne separation function. Testing is performed under the rigorous conditions posed by randomly generated, highly constrained traffic scenarios. The test environment selected for this initial phase is a generic circular scenario with random trajectories and all flight paths (including conflict resolution paths) restricted to a single altitude. The random traffic scenarios are generated such that they exhibit a high rate of conflicts whose lateral encounter angles vary over nearly the full range of values.

Subsequent phases of the SPAS study will be expanded to include three-dimensional (3D) airspace, and traffic structures will be constrained to resemble real traffic patterns found in the NAS. These multi-flight-level scenarios will exhibit aircraft conflict geometries more like those found in real traffic conditions, but they will be modified to increase the traffic density and conflict frequency. Through the incremental incorporation of various errors, uncertainties, delays, and other variables, and through the testing of ASAS capabilities designed to compensate for these factors, the complete SPAS study will provide some of the key data needed for a comprehensive safety analysis of airborne separation concepts and for comparison to other methods of separation management.

\section{Background}

Safety evaluation of new Air Traffic Management concepts and technologies involves modeling the often very complex relationships between individual subsystems in order to assess system reliability and robustness. The effect of subsystem failures or degradation on the system performance needs to be assessed by analytical tools such as fault tree analyses and other risk assessment techniques that require quantitative safety data as input ${ }^{3,4}$. The overall goal and scope of the SPAS study is to begin to understand and quantify some safety performance aspects of ASAS applications using system uncertainties and prediction error models that will eventually contribute to the safety analysis of NextGen.

Until now, safety evaluation of ASAS applications have for the most part been based on low fidelity studies, seldom including assessment of system uncertainties. One such study ${ }^{5}$ addressed the "feasibility" of two conflict detection and resolution methods in a quantitative simulation study that measured safety, efficiency and stability of the system. Safety was evaluated in terms of the ability of the two methods to successfully resolve all conflicts in the airspace. The study emphasized that both ASAS methods resolved all conflicts with only small path deviations and 
that safety was not compromised. An additional stability metric reflected the potential propagation of conflicts as a result of decentralized resolution methods. The study posed that lack of stability would represent an efficiency degradation if the number of path deviations is increased as a result of additional conflict resolutions. The opposite was observed in the study, which concluded that both methods provide very efficient resolutions. A later study ${ }^{6}$, intending to compare the performance of centralized and decentralized air traffic separation methods, revisited the notion of stability and efficiency metrics in a low fidelity model of three separation techniques.

The safety of ground-based automated separation was the subject of a recent study ${ }^{7}$ that presented a preliminary fault-tree analysis of the effect of some system faults on the safety of a centralized separation system. The analysis did not consider system uncertainties or performance degradation, nor did it consider prediction errors. Based on assumed fault rates, the study suggested that system safety would be preserved. In particular, it concluded that under the assumptions made, the system may be able to achieve safety levels higher than today's levels.

\section{Simulation Platform and Scenarios Description}

\section{A. The Airspace \& Traffic Operations Simulation}

The SPAS experiments are being conducted at the Air Traffic Operations Laboratory (ATOL) at NASA Langley Research Center utilizing a distributed simulation platform that includes a grid of aircraft simulators interconnected through a High Level Architecture communication infrastructure. The simulation platform, known as the Airspace \& Traffic Operations Simulation $(\text { ATOS })^{8}$ depicted in Fig. 1, can be used for batch Monte Carlo studies as well as real time human-in

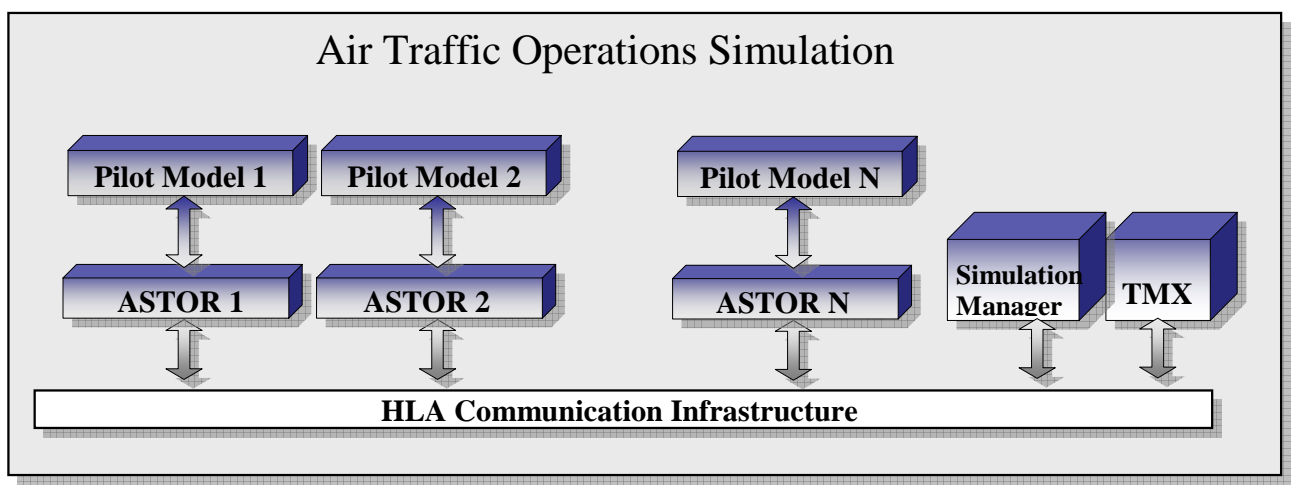

Figure 1. SPAS Simulation Platform

the-loop experiments. The ATOS is comprised of a number of real time, medium fidelity aircraft simulators referred to as Aircraft Simulation for Traffic Operations Research (ASTOR), and a Simulation Manager. Each ASTOR represents a single aircraft and is modeled by a 6-degree of freedom dynamic model, a Flight Management System (FMS), Mode S Automatic Dependent Surveillance - Broadcast (ADS-B) datalink capability, and a prototype airborne separation automation that is described in the next section. For batch simulations, all ASTORS are "flown" by a computer-based pilot model (PM) that performs the basic pilot actions for airborne conflict management. The PM was designed to automatically take over the controls of an ASTOR with no interference to the system and without the aid of a human pilot. The PM design is composed of three parts: a sensory input model, a rule-based decision model, and an actuator response model. The sensory input model gathers information and passes it to the rules model. The rules model determines the PM actions and commands the pilot action model to execute that action. Both the sensory input and actuator response models are governed by a simple time delay algorithm. A normally distributed time delay is used to configure the PM "personality" as one of several profiles which dictates its responsiveness level. The preliminary baseline experiment uses a "normal" PM with a mean and maximum sensory delay time 500 and 5000 milliseconds respectively.

Other ATOS modules include a background traffic generator capability called TMX ${ }^{9}$ that models lower fidelity air traffic with full airborne separation capabilities and provides a plan view display for traffic visualization. The communication infrastructure allows the ATOS capabilities to be extended by connecting with other real time or batch simulation environments. 


\section{B. Airborne Conflict Management System}

The intent of this study is to document whether adequate safety performance of airborne separation is achievable without regard to the optimality of the specific ASAS approach and algorithms used. A brief description of the system chosen for the study follows.

The airborne conflict management system used in this experiment series is the Autonomous Operations Planner (AOP), a NASA-developed research model of an airborne automation system built for the study of advanced distributed air-ground operational concepts ${ }^{10}$. The AOP provides an integrated suite of capabilities for managing traffic conflicts and trajectory changes from the flight deck perspective, including conflict detection, resolution, prevention, and trajectory constraint conformance. These capabilities are developed to a level sufficient for research of complete airborne responsibility for self-separation as defined by the Principles of Operation of ASAS, Category $4^{2}$. In the JPDO NextGen Concept of Operations, they correspond to the functions of Separation Management and aspects of Trajectory Management ${ }^{1}$.

The intent-based conflict detection (CD) function of AOP uses state and intent data received from other traffic aircraft over ADS-B in combination with ownship state data, autoflight mode settings, and flight plan information to deterministically predict future losses of separation. For this study, the autoflight mode remained coupled to the FMS for lateral navigation. The CD look-ahead horizon was set to 10 minutes and the separation criterion to 5.2 nautical miles. A capability exists in AOP to apply CD buffers tailored to individual flight segments to account for trajectory prediction error sources such as wind-field forecasts ${ }^{11}$. Although the baseline runs of this preliminary study excluded most error sources and corresponding buffers, they are planned for inclusion in upcoming stages of the experiment series. AOP also has a second, independent, CD system that uses state-vector projections to detect flight crew blunders and prediction faults of the intent-based CD system. A complete system is envisioned to contain both state-based and intent-based CD systems for redundancy and safety ${ }^{12}$. Conflict alerting is modeled after the multi-alert-level approach recommended by RTCA ${ }^{13}$. The timing of alerts between aircraft are staggered as a method for incorporating a right-of-way rule set (i.e. priority rules), based roughly on the set used in Visual Flight Rules $^{14}$. In the current study, the simple computer-based pilot model reacted to "low level alerts" within a normally distributed timing scheme defined by response type. When alerted to a conflict, the pilot model's action was to request a fully-conflict-free resolution trajectory from AOP.

For conflict resolution (CR), AOP contains both strategic and tactical capabilities. Tactical CR refers to openloop vectors or altitude changes to solve conflicts with no predetermined reconnection plan. Along with the statebased CD, the tactical CR capability was disabled to allow the focus of the current study to be on distributed strategic systems. Strategic CR refers to the single action of modifying the flight plan such that the conflict is solved and the aircraft reconnects to the previous trajectory. In the current study, strategic CR solutions were purposefully constrained to lateral maneuvers only. In generating solutions, AOP's strategic CR system takes into account all known trajectory constraints, including trajectories of nearby traffic, airspace hazards, ownship performance limits, and required time of arrival (RTA) constraints. A genetic algorithm is employed to search within a set of predefined geometric patterns to generate CR trajectories that simultaneously accommodate these constraints ${ }^{15}$. Once a suitable CR trajectory is calculated, and it is verified conflict free for 20 minutes based on current traffic data (this time is not fully applicable to the current traffic scenario because new aircraft are continuously being created during the test runs), it is presented to the flight crew for review. In the current study, the pilot model was programmed to execute the first available CR trajectory. Immediately upon execution of the route, ADS-B broadcasts the new ownship intent as a series of trajectory change points.

AOP also includes functions for conflict prevention, including at-a-glance maneuver restriction symbology for the flight crew and support for tactical / strategic trajectory probing (also known as provisional CD). These functions were not required for this study and were therefore disabled. 


\section{Experiment Design}

A real time scenario generation tool was developed for this experiment that creates initially straight, random aircraft routes traversing a circular test area representing a generic, high density, en route sector. In this environment, ASTOR aircraft are introduced into the scenario on the random routes, traverse the airspace while detecting and solving conflicts, and exit the airspace while proceeding in the direction of the initial downstream waypoint constrained by a RTA. A conflict is defined as a predicted Loss of Separation (LOS) between two aircraft. The applicable separation standard used is $5 \mathrm{NM}$. After leaving the test area and before reaching the RTA waypoint, metrics for the flight are recorded and the ASTOR is reinitialized with a new random route and reintroduced to the test area. The number of independent ASTOR aircraft (i.e. each a separate networked computer) operating simultaneously in the simulation ranged from 14 to 72 .

The test area, depicted in Figure 2 , is modeled as two concentric circles of $80 \mathrm{NM}$ and $160 \mathrm{NM}$ radii, respectively. The region between the circles is referred to as the initialization region, and the region within the inner circle is the test region. All aircraft are created just outside the initialization region and their paths traverse the test region where experiment metrics are collected. Because AOP functions were purposefully disabled in the initialization ring,

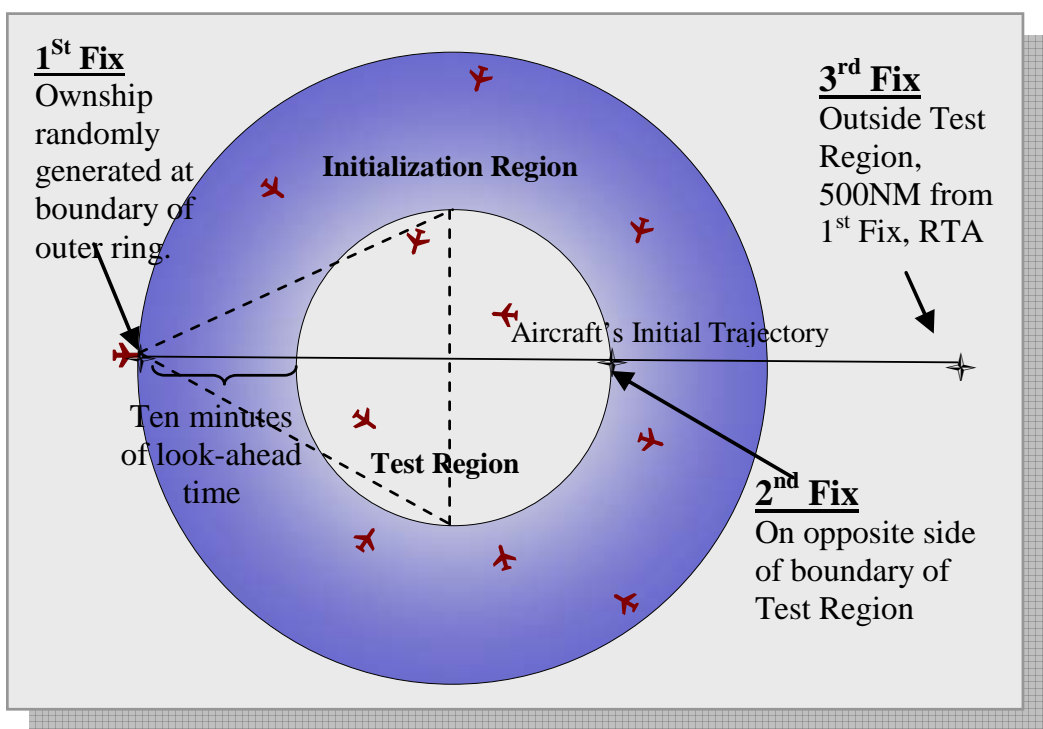

Figure 2. Scenario Design conflicts fully outside the test region were excluded from analysis, as were LOS events where either aircraft was outside the test region. The relative dimensions of the two circles were chosen to allow approximately 10 minutes of look-ahead time for the strategic conflict detection and 20 minutes of flying time in the test region. The target traffic demand was maintained within the limitations of the simulation platform, typically with a standard deviation of \pm 3 aircraft or less.

The initial route was defined by three fixes. The first fix was generated as a [latitude, longitude] pair randomly placed on the perimeter of the outer circle. The second fix, located randomly on the opposite side of the inner circle and also defined as a [latitude, longitude] pair, was chosen to ensure that all initial trajectories intersect the test region. For all newly initiated aircraft entering the initialization region, the scenario generation logic ensured that their respective initial positions were at least $10 \mathrm{NM}$ apart and that their respective paths would not intersect in this region. As stated earlier, any conflicts occurring fully outside the test region were ignored and not solved. The third fix was created outside the test region, approximately $500 \mathrm{NM}$ from the first fix along the straight line route created by the first and second fixes. An RTA constraint was placed on the third fix to represent flow-managed operations. Conflict resolutions were required to maintain RTA conformance when possible. All trajectories were placed at the same altitude and only lateral resolutions were exercised in order to maintain the desired traffic densities. As mentioned above, all aircraft were terminated as soon as they exited the test region and reinitialized for reinsertion on a new random route.

Table 1. Traffic Demand for Busiest Altitude in Sectors ZOA31 and ZOB46 on 19 Feb 2004

\begin{tabular}{|c|c|c|c|c|}
\hline & \multicolumn{2}{|c|}{$\begin{array}{c}\text { Median Sector } \\
\text { (ZOA31) } 16,624 \text { sq NM } \\
\text { (Oakland) }\end{array}$} & \multicolumn{2}{|c|}{$\begin{array}{c}\text { Dense Sector } \\
\text { (ZOB46) 5,959 sq NM } \\
\text { (Cleveland) }\end{array}$} \\
\hline & Mean & Peak & Mean & Peak \\
\hline Sector density at FL310 & 3 & 5 & 5 & 10 \\
\hline $\begin{array}{r}\text { Normalized } \\
1 X \text { Density per } 10000 \mathrm{NM}^{2}\end{array}$ & 1.8 & 3.0 & 8.45 & 16.85 \\
\hline
\end{tabular}


Traffic demand levels in the NAS were analyzed using ETMS flight data from 19 February 2004, the good weather, high-traffic day selected by the JPDO and the FAA to represent baseline " $1 \mathrm{X}$ " density. An analysis using the NASA ACES ${ }^{16}$ analysis tool was conducted to determine the traffic count for every high altitude sector in the United States, at each flight level, for the 24 hour period. From this data, the mean and maximum traffic count for FL310 (the busiest altitude within the selected sectors) was determined for the median sector ZOA31 and a very busy en route sector ZOB46. Table 1 shows the " $1 X$ " traffic demand from 19 February 2004 in these two sectors: ZOA31, typical traffic density and similar in size to the test scenario, and ZOB46, a much smaller sector with very dense and complex traffic conditions. The traffic levels were then normalized to produce densities based on $10,000 \mathrm{NM}^{2}$.

\section{Experiment Results}

\section{A. Input Data: Analysis of the Conditions Produced in the Test Scenarios}

The experiment consisted of six sets of simulation runs as indicated in Table 2. Each run was six hours of continuous simulation at the sustained traffic density level. Each run set consisted of six independent replicates of the six-hour run, except for the two highest density conditions, which had only two replicates each.

Table 2: Summary of Simulation Runs

\begin{tabular}{|c|c|c|c|c|c|c|c|}
\hline $\begin{array}{c}\text { Run } \\
\text { Set }\end{array}$ & $\begin{array}{c}\text { Sustained } \\
\text { Mean } \\
\text { Density per } \\
10000 \mathrm{NM}^{2}\end{array}$ & $\begin{array}{c}\text { Standard } \\
\text { Deviation }\end{array}$ & $\begin{array}{c}\text { Peak } \\
\text { Density per } \\
10000 \mathrm{NM}^{2}\end{array}$ & $\begin{array}{c}\text { Total } \\
\text { Number of } \\
\text { Simulation } \\
\text { Hours }\end{array}$ & $\begin{array}{c}\text { Total } \\
\text { Number } \\
\text { of Flights }\end{array}$ & $\begin{array}{c}\text { Total } \\
\text { Number } \\
\text { of Flight } \\
\text { Hours }\end{array}$ & $\begin{array}{c}\text { Total } \\
\text { Number } \\
\text { of } \\
\text { Conflicts }\end{array}$ \\
\hline 1 & 3.45 & 0.59 & 4.97 & 36 & 881 & 237.27 & 195 \\
\hline 2 & 6.11 & 0.83 & 8.29 & 36 & 1527 & 418.6 & 550 \\
\hline 3 & 8.61 & 0.97 & 11.44 & 36 & 2195 & 544.57 & 1018 \\
\hline 4 & 11.64 & 1.23 & 15.34 & 36 & 3000 & 797.17 & 1788 \\
\hline 5 & 15.24 & 1.49 & 19.31 & 12 & 1302 & 347.45 & 963 \\
\hline 6 & 17.18 & 1.54 & 21.39 & 12 & 1560 & 399.08 & 1256 \\
\hline Totals
\end{tabular}

The peak and sustained mean density for each replicate was computed and normalized to $10000 \mathrm{NM}^{2}$. As can be seen, the sustained traffic densities for the different sets either match or surpass the values indicated in Table 1 . The table also includes the total number of simulation hours, unique generated flights, cumulative flight hours and total number of conflicts for each density set.

The mean number of traffic conflicts generated within the test region per flight hour as a function of normalized traffic density is shown in Fig. 3. As the graph shows, the frequency of conflicts per flight hour increases with traffic density ranging from an average of 0.82 conflicts per flight hour for a density of 3.45 aircraft per $10000 \mathrm{NM}^{2}$ to 3.15 conflicts per flight hour for densities of 17.8 aircraft per $10000 \mathrm{NM}^{2}$. The high frequency of conflicts is a result of the highly constrained, randomly generated traffic scenarios.

Correlation of this metric with the number of potential conflicts predicted

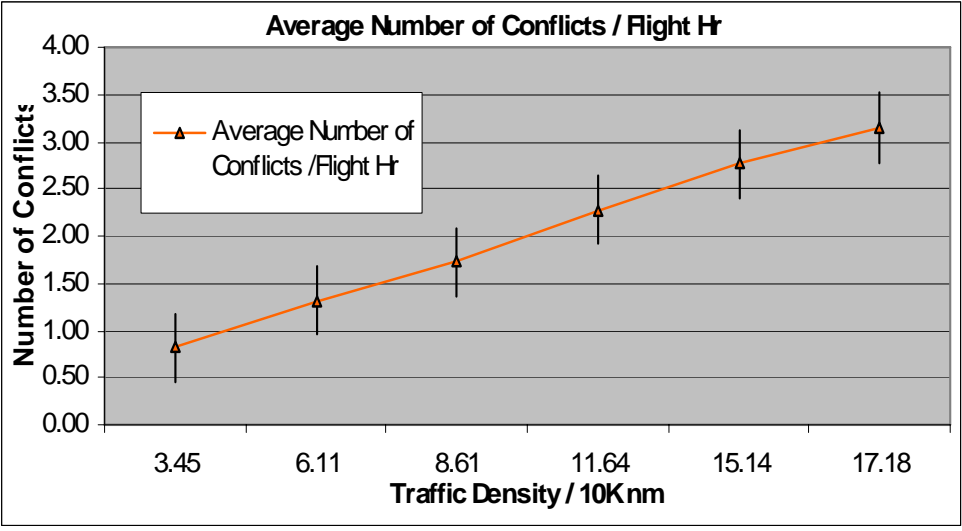

Figure 3. Conflict Count Per Flight Hour in real air traffic conditions is not easy since the data is not readily available. The frequency of predicted conflicts could be loosely equated to the number of times route or speed adjustments are issued to aircraft to avoid potential separation violations. Controller instructions are communicated via voice, making a comparison metric difficult to 
retrieve. A study conducted to develop air traffic scenarios resembling real traffic conflict conditions using Host Computer System tracks of the Memphis Air Route Traffic Control Center (ARTCC) estimated a total of 203 predicted conflicts for 4332 flight hours ${ }^{17}$. That would represent about 0.05 conflicts per flight hour for an estimated mean traffic density of 2 to 4 aircraft per $10000 \mathrm{NM}^{2}$ (at the densest flight level in the ARTCC).

Another important property used to characterize the conflict traffic scenarios is the encounter angle distribution. The conflict encounter angles observed in the random traffic scenarios used in this experiment are depicted in Figure 3.. The graph shows that the proportion of conflict angles is maintained within each group for all tested densities. The percentage of small angle (0 to 30 degrees) conflicts is less than all other angle brackets. However, the actual count of low angle conflicts observed is 163 per 1000 flight hours as compared to 14 small angle conflicts per 1000 flight hours observed in the extracted

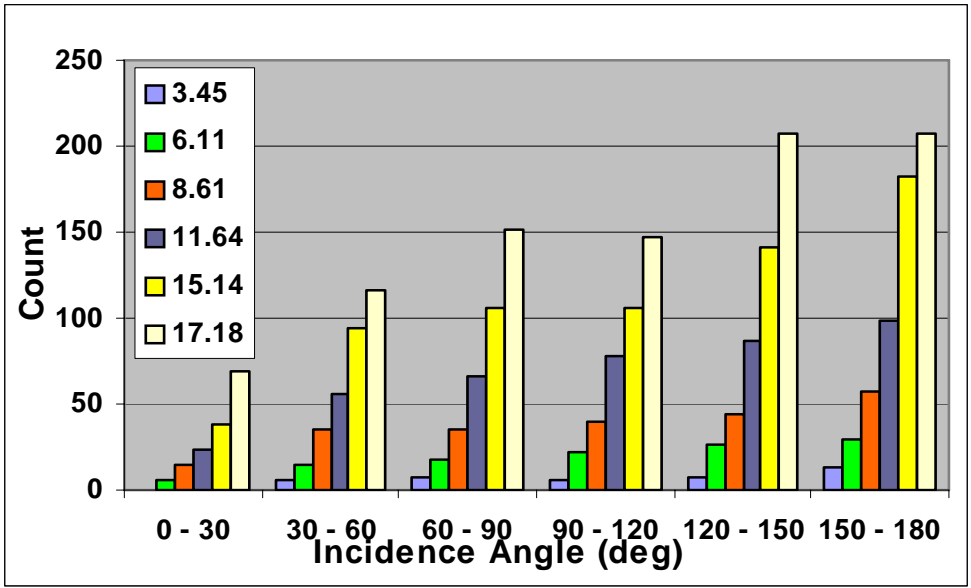

Figure 4 Conflict Encounter Angle scenarios reported in the study above mentioned ${ }^{17}$. The Memphis ARTCC study, which did not involve random routing, indicates that almost 30\% (approximately 60 conflicts) of all conflicts angles are between 0 and 30 degrees, which are considered the most challenging class of conflicts, and less than $10 \%$ are between 120 and 150 degrees. All other angle brackets are evenly represented in the data. In the current experiment, low angle conflict frequency was reduced as a result of measures taken to minimize conflicts in the initialization ring.

The predicted separation at the closest point of approach (CPA) gives a measure of the magnitude of the predicted intrusions and the complexity of the traffic scenarios. Figure shows the distribution of horizontal distances at the predicted CPA for each traffic density. The distances are roughly evenly distributed between 0 and 5 NM for low traffic densities. As traffic density increases (and conflict count increases), predicted CPAs are more prevalent between 3 and $5 \mathrm{NM}$, which is reasonable considering the greater area bounded by these radii and therefore the greater probability of containing a predicted CPA. A small number of conflicts were recorded between 5 and 5.1 NM as a result of a conflict detection buffer.

\section{B. Output Data: Analysis of the Experiment Outcomes}

The conflicts shown in Fig. 5 were detected and resolved using the technique of distributed airborne separation. Figure 6 presents the distribution of actual CPAs (i.e. as flown after resolution maneuvers were implemented) for these same aircraft pairs. Non-conflicting aircraft pairs were not included in this data. The data show that all conflicts were solved, with the exception of three borderline cases. The CPAs of these cases were 4.986, 4.989, and 4.999 NM (i.e. maximum protected zone penetration of 85 feet). In two of these cases, an aircraft was executing a turn at the time of LOS. These two borderline conflicts were not detected as a result of modeling the turns as a 
series of straight segments, whereas the ASTOR flies curved turns. In addition, all prediction buffers were set to zero for this baseline study. The cause of the third borderline LOS (4.989) could not be precisely determined but may have also involved a turn modeling issue. In all three cases, the use of prediction buffers and/or tactical CR capability would have prevented the borderline penetrations. The distribution of actual CPAs describes the effectiveness of the ASAS system and the constraints posed by the traffic density.

The CPA distributions show that many aircraft end up being separated by much more than the minimum requirement of $5 \mathrm{NM}$. This result reflects the notion of a busy airspace, in which a given aircraft that resolves a conflict may subsequently encounter multiple additional conflicts at later times. The end result can be multiple resolution maneuvers that further separate the initial conflicting pair. As traffic density increases, a greater spread in the CPA distribution is observed. For example, at the lowest traffic density condition, few CPAs were recorded beyond $11 \mathrm{NM}$. At higher traffic density conditions, a number of large separation distances (beyond $11 \mathrm{NM}$ ) are recorded, as well as an increase close to the minimum value of 5 NM. Distribution of distances shows a marked "peak" between 5 and $7 \mathrm{NM}$ suggesting that as the airspace gets saturated, aircraft are constrained to fly closer to the separation minima. At lower densities there seems to be a more uniform spread of values.

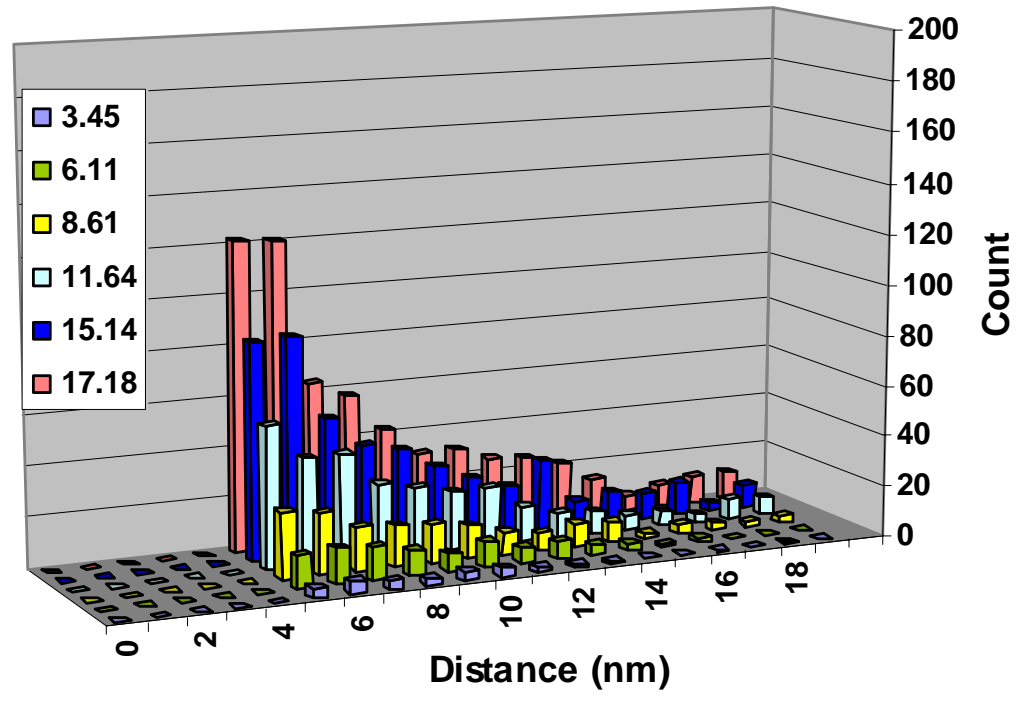

Figure 6. Actual Distances at the CPA

The median conflict alert duration is shown in Figure . It represents the time betwee conflict detection and the time the conflict alert cleared, i.e. the conflict was resolved. This metric reflects the effectiveness of the resolution automation and the constraints imposed by traffic density in finding suitable resolutions. The increasing durations follow the increasing traffic density.

Conflicts were classified as first generation and second generation, where a second generation conflict is one that is created as a result of solving a previous conflict. This is referred to as "secondary conflicts" in the studies referenced earlier 5,6 . Second generation conflicts are further classified based on the conditions preceding the conflicts. In particular, the so-called "sidewalk" and "coincidence" conflicts were investigated in this experiment. Repeated conflicts between the same pair of aircraft, caused by simultaneous resolutions of previous conflicts are

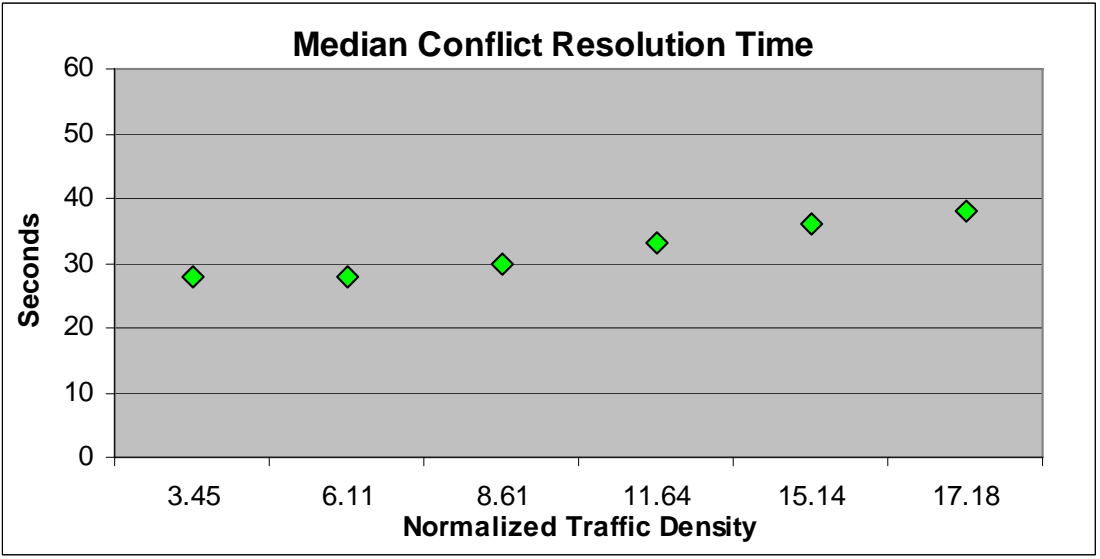

Figure 7. Median Conflict Alert Duration identified as sidewalk

conflicts. Coincidence conflicts are the result of two aircraft simultaneously solving unrelated conflicts. These conditions, which are the result of distributed decision making, seem to have a very low probability, even in the rigorous laboratory conditions of this experiment. Out of nearly 2800 simulated flight hours and nearly 6000 conflicts in this study, only 11 conflicts were identified as possible coincidence conflicts and no sidewalk conflcits 
were observed. With initial detection occurring near 10 minutes from predicted LOS, all of these cases were safely resolved.

\section{Future Work}

As mentioned earlier, this experiment is the first of a series of studies designed to analyze and evaluate the safety performance of airborne separation. The next set of studies will seek to evaluate safety performance in similar simulation conditions introducing one element of uncertainty at a time to understand its individual effect. A set of exploratory runs were performed to begin the design of this next set of experiments. The initial observations were compared with the baseline run described in this paper in which no prediction errors or uncertainties were modeled. This means ADS-B reception was perfect, full intent was shared, the pilot model responded at "normal" rates, and wind predictions were accurate. The exploratory runs included the following configuration settings and were based in all cases on a mean traffic density of 8.61 aircraft per 10000 NM2 (about 170 flights over a four hour run) and an average of 46 flight hours. The following conditions were individually tested.

1. Pilot Model: The pilot model was configured to have a sensorial delay so that the reaction time to a conflict alert was longer than the "normal" case. The "sleepy" sensor delay mean and standard deviation used were 2000 and 2.0 milliseconds respectively.

2. ADS_B signal interference: The ADS-B model was configured to have default signal degradation settings due to interference and distance as described in ${ }^{18,19}$

3. ADS-B intent exchange: Two runs, one sending four trajectory change points (TCP) and one sending two TCPs for intent transmission were performed. Typical CR maneuvers require three to four TCPs to define the complete maneuver..

4. Wind prediction error: Four runs were performed to test a modest wind speed prediction error of $10 \mathrm{NM} / \mathrm{hour}$. For the first two runs, no attempt was made to compensate for the resulting trajectory prediction uncertainty. For the second set of runs, a longitudinal buffer was used to increase the likelihood of conflict detection.

A set of metrics were collected to begin to probe the system's safety performance in the presence of these limitations and uncertainties. The graph in Figure 3 shows the observed effect on conflict counts relative to the baseline. No statistics were possible given the limited amount of data collected. The percentage of change in the observed number of conflicts in the PM and 4-TCP runs seems to indicate little effect. The ADS-B interference and 2-TCP runs exhibit an observed increase in conflict detections of about $20 \%$. In all four runs there were no losses of separation.

The first set of wind prediction error runs showed a slight increase in the number of observed conflicts detections. In addition, there were three losses of separation, with CPAs of 4.94, 4.98 and 4.90 NM. During the second set wind prediction error runs that employed longitudinal buffers, the observed number of conflict detections were higher, but there were no losses of separation. This is just a small sample of on-going research;

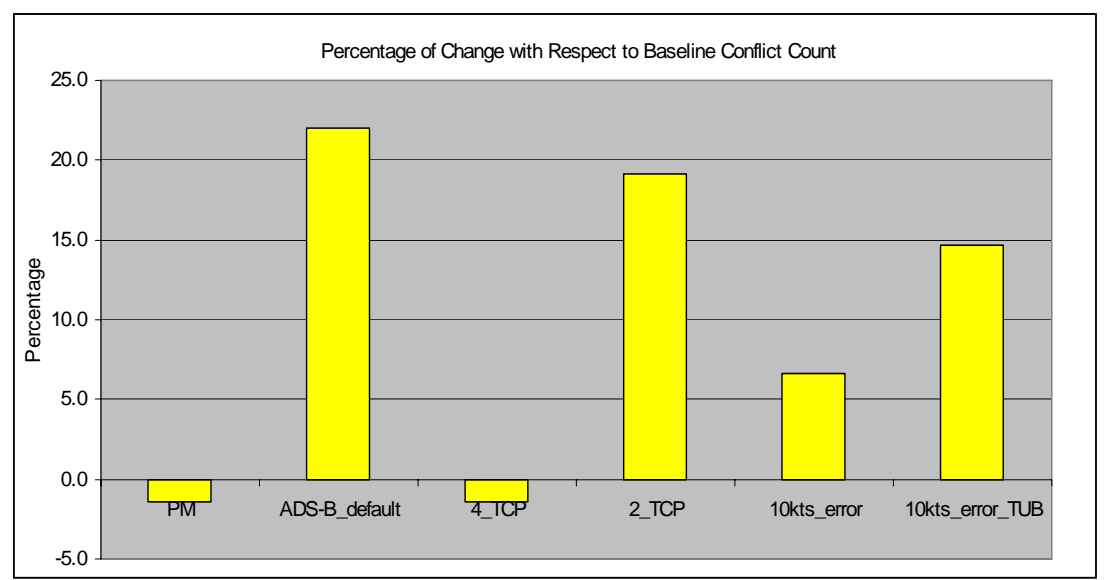

Figure 3. Exploratory Runs many more exploratory runs will be conducted to guide the next set of experiments in the SPAS study.

\section{Conclusion}

The SPAS experiment suite is a methodical, rigorous, and incremental approach to the study of the safety implications of prediction errors and system uncertainties on airborne separation. This study is unique in that it provides new research results based on medium fidelity simulation systems, with key sub-systems such as the ASAS 
and ADS-B modeled in high fidelity. Early results at five times the typical traffic density in today's NAS indicate that a distributed approach to maintaining separation of aircraft can be safely achieved, even after introducing several sources of error and uncertainty. Research scheduled within the next two years, examining higher levels of density, multiple altitudes, other sources of error and uncertainty, and coordinating with a centralized approach, will add greatly to the knowledge of methodologies to safely automate the separation of aircraft.

\section{Acknowledgments}

The research team acknowledges the indispensable work by our ATOS simulation development team Daniel Finkelsztein, John Bunell, Doug Mielke, Robert Vivona, David Karr, David Roscoe, and Frank Bussink, and valued assistance from Ed Scearce, Jeff Viken, Jeremy Smith, Dave McNally, and Mike Paglione.

\section{References}

${ }^{1}$ Joint Planning and Development Office: Concept of Operations for the Next Generation Air Transportation System.”, Version 2.0, June 2007. http://www.jpdo.gov/library/NextGen_v2.0.pdf

${ }^{2}$ FAA/Eurocontrol Cooperative R\&D Action Plan 1, "Principles of Operation for the Use of Airborne Separation Assurance Systems.”, Version 7.1, June 2001. http://www.eurocontrol.int/care-asas/gallery/content/public/docs/po-asas71.pdf

${ }^{3}$ FAA System Safety Handbook, Chapter 9: Analysis Techniques, December 30, 2000

${ }^{4}$ Stephens, Richard, A. and Talso, Warner, System safety Analysis Handbook: A Source Book for Safety Practitioners, System, Safety Society, 2nd Edition, August 1999.

${ }^{5}$ Bilimoria,K., Sheth, H., Grabble, S., "Performance Evaluation of Airborne Separation Assurance for Free Flight,” AIAA Guidance, Navigation, and Control Conference and Exhibit, AIAA 2000-4269, August 2000

${ }^{6}$ Krozel, J., Peters, M., Bilimoria, K., Lee, C., and Mitchell, J., "Systems Performance Characteristics of Centralized and Decentralized Air traffic Separation Strategies,” $4^{\text {th }}$. USA/Europe Air Traffic Management R7D Seminar, Santa Fe, NM, December 2001.

${ }^{7}$ Andrews, J., Erzberger, H., and Welch, J., “Safety Analysis for Advanced Separation Concepts,” Air Traffic Control. Quarterly, Vol. 14, No. 1, 2006, pp. 5-24

${ }^{8}$ Finkelstein, D., Lung, Vivona, R., Bunell, J., Mielke D., and Chung W.: “Airspace and Traffic Operations Simulation for Distributed ATM Research and Development.” AIAA 2005-6488.

${ }^{9}$ Bussink, Hoekstra, and Heesbeen: “Traffic Manager: A Flexible Desktop Simulation Tool Enabling Future ATM Research.” DASC 2005, pp. 3.B.4-1 to 3.B.4-10.

${ }^{10}$ Karr, D., Roscoe, D., and Vivona R., “An Integrated Flight-Deck Decision-Support Tool in an Autonomous Flight Simulation,” AIAA Modeling and Simulation Technologies Conference and Exhibit, AIAA-2004-5261, August 2004

${ }^{11}$ Karr, D., Roscoe, D., and Vivona R., "Conflict Detection Using Variable 4D Uncertainty Bounds to Control Missed Alerts,” AIAA Guidance, Navigation, and Control Conference and Exhibit, AIAA-2006-6057, August 2006

${ }^{12}$ Wing, D., Barmore, B., and Krishnamurthy, K., "Use of Traffic Intent Information by Autonomous Aircraft in Constrained Operations,” AIAA Guidance, Navigation, and Control Conference and Exhibit, AIAA-2002-4555, August 2002.

${ }^{13}$ RTCA Special Committee 186: "Application of Airborne Conflict Management: Detection, Prevention, \& Resolution.” RTCA/DO-263, December 2000.

${ }^{14}$ Wing, D.J., Krishnamurthy, K., Barhydt, R., and Barmore, B., "Pilot Interactions in an Over-constrained Conflict Scenario as Studied in a Piloted Simulation of Autonomous Aircraft Operations,” 5th USA/Europe Air Traffic Management R\&D Seminar (ATM2003), Budapest, Hungary, June 2003.

${ }^{15}$ Vivona, R., Karr, D., and Roscoe, "Pattern-Based Genetic Algorithm for Airborne Conflict Resolution,” AIAA Guidance, Navigation, and Control Conference and Exhibit, AIAA-2006-6060, August 2006

16 Jesse Aronson†, Vikram Manikonda*, Wilbur Peng*, Renato Levy* and Karlin Roth‡, “An HLA Compliant Agent-Based Fast-Time Simulation Architecture For Analysis Of Civil Aviation Concepts,” Spring SISO Simulation Interoperability Workshop, April, 2003

${ }^{17}$ Paglione, M., Oaks, R. and Bilimoria, K., "Methodology for Generating Conflcit Scenarios by Time Shifting Recorded Traffic Data,” AIAA $3^{\text {rd }}$. Aviation Technology , Integration and Operations Conference, AIAA 2003-6724, Denver, Colorado, November 20003

${ }^{18}$ Chung, W. and Staab, R. “A 1090 Extended Squitter Automatic Dependent Surveillance - Broadcast (ADS-B) Reception Model for Air-Traffic-Management Simulations,” AIAA 2006-6614, Modeling and Simulation Technologies Conference and Exhibit, August 2006,

${ }^{19}$ Barhydt, R., Palmer, M., Chung, W., and Loveness, G., “ADS-B within a multi-aircraft simulation for distributed airground traffic management,” Digital Avionics Systems Conference (DASC), 2004. 